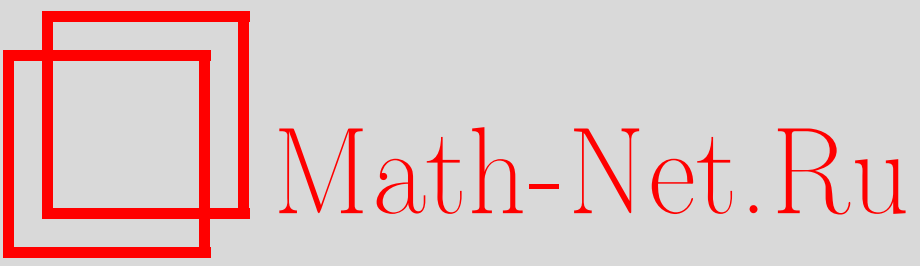

Я. Л. Цеслински, Геометрия подмногообразий, полученных из Spin-значных спектральных задач, ТМФ, 2003, том 137, номер 1, 47-58

DOI: https://doi.org/10.4213/tmf244

Использование Общероссийского математического портала Math-Net.Ru подразумевает, что вы прочитали и согласны с пользовательским соглашением

http://www.mathnet.ru/rus/agreement

Параметры загрузки:

IP: 52.205.19.152

26 апреля 2023 г., 06:04:47 


\section{ГЕОМЕТРИЯ ПОДМНОГООБРАЗИЙ, ПОЛУЧЕННЫХ ИЗ Spin-ЗНАЧНЫХ СПЕКТРАЛЬНЫХ ЗАДАЧ}

Представлены результаты, мотивированные теорией Сима солитонных поверхностей. Некоторые конкретные классы поверхностей могут быть получены исходя из достаточно общих предположений о структуре спектральной задачи. Новым и неожиданным моментом является то, что в ряде случаев (включая псевдосферические поверхности) этот подход не зависит от выбора координат. Преобразование Дарбу-Беклунда формулируется в терминах чисел Клиффорда, что значительно упрощает построение явных решений: громоздкие вычисления в матричных представлениях заменяются представлениями вращений элементами подходящей группы Spin. Наконец, спектральная задача и спектральный параметр допускают чисто геометрическое описание в случае изометрических погружений пространств постоянной кривизны в сферы и евклидовы пространства.

Ключевые слова: солитонные поверхности, преобразование Дарбу-Беклунда, алгебра Клиффорда, группа Spin.

\section{1. ПОДХОД СОЛИТОННЫХ ПОВЕРХНОСТЕЙ}

Рассмотрим класс спектральных (линейных) задач вида

$$
\Psi, k=U_{k}(\lambda) \Psi, \quad k=1, \ldots, m
$$

где $U_{k}$ - матрищы, зависящие от $x^{1}, \ldots, x^{m}$ и $\lambda, \Psi$ - некоторая матрица (фундаментальное решение), а запятая означает дифференцирование $\left(\Psi_{,}:=\partial \Psi / \partial x^{k}\right)$.

Основная идея развитого Симом подхода солитонных поверхностей [1], [2] заключается в том, чтобы сопоставить этой спектральной задаче некоторый класс поверхностей с помошью так называемой формулы Сима-Тафеля

$$
F:=\Psi^{-1} \frac{\partial \Psi}{\partial \lambda}
$$

Отметим следующие моменты:

*Instytut Fizyki Teoretycznej, Uniwersytet w Białymstoku, ul. Lipowa 41, 15-424 Bialystok, Poland. E-mail: janek@alpha.uwb.edu.pl 
1. Матрища $F$ зависит от $x^{1}, \ldots, x^{m}$ и $\lambda$. Рассматривая эту матрищу как элемент некоторого линейного пространства, можно отождествить ее (при любом фиксированном $\lambda$ ) с погружением области в $\mathbb{R}^{m}$ в это линейное пространство. Таким образом, $F$ описывает $\lambda$-семейство $m$-мерных подмногообразий.

2 . Если $\Psi$ принимает значения в группе Ли $G$, то $F$ является $\lambda$-семейством погружений в соответствуюшую алгебру Ли.

3. Спектральные задачи в $s u(2)$ (в том числе, класс Захарова-Шабата-АбловицаКаупа-Ньюэлла-Сигура (ЗШ-АКНС) пар Лакса) приводят к поверхностям, погруженным в $\mathbb{R}^{3}$.

Во многих случаях полученные с помощью формулы Сима "солитонные поверхности" допускают удобную геометрическую интерпретацию, что будет продемонстрировано в данной статье. Подход солитонных поверхностей успешно применяется при изучении псевдосферических поверхностей, поверхностей постоянной средней кривизны, поверхностей положительной гауссовой кривизны, поверхностей Бианки, вихревых нитей (локализованного уравнения индукции), изотермических поверхностей, ортогональных сетей, сетей Гишара и др. [2]-[4].

Основная цель данной статьи - представить новые результаты, полученные в рамках подхода солитонных поверхностей. В частности, подчеркивается и разъясняется роль групп Spin. Доказательства и детали вычислений, как правило, опускаются. Часть из них имеется в цитированной литературе, часть будет опубликована отдельно.

\section{2. ПСЕВДОСФЕРИЧЕСКИЕ ПОВЕРХНОСТИ}

Рассмотрим линейную задачу

$$
\begin{aligned}
& \Psi,,_{x}=U \Psi, \quad U:=A \mathbf{e}_{1}+\lambda\left(B \mathbf{e}_{2}+C \mathbf{e}_{3}\right), \\
& \Psi,_{y}=V \Psi, \quad V:=P \mathbf{e}_{1}+\frac{1}{\lambda}\left(Q \mathbf{e}_{2}+R \mathbf{e}_{3}\right),
\end{aligned}
$$

где $A, B, C, P, Q$ и $R$-некоторые скалярные функции $x$ и $y, \mathbf{e}_{1}, \mathbf{e}_{2}, \mathbf{e}_{3}$ - ортонормированньй базис в $s u(2)$ (например, $\left.\mathbf{e}_{k}:=-i \sigma_{k} / 2\right), \Psi=\Psi(x, y ; \lambda)$ (фундаментальное решение) принимает значения в $S U(2)$ и нет никаких других ограничений (за исключением условий согласования).

Теорема 1 [5]. Пусть $F=\Psi^{-1} \Psi, \lambda$, где $\Psi$ удовлетворяет задаче (3). Тогда:

а) $F=f_{1} \mathbf{e}_{1}+f_{2} \mathbf{e}_{2}+f_{3} \mathbf{e}_{3}$, әде $f_{k}-$ вещественнье функции $x, y$ и $\lambda$;

б) $\left(f_{1}, f_{2}, f_{3}\right)$ - $\lambda$-семейство псевдосферических погружений в $\mathbb{R}^{3}$, т.е. гауссова кривизна поверхности $F$ постоянна и отрицательна: $K=-\lambda^{2}$;

в) $x$ и у являются асимптотическими координатами (координатами светового конуса в физической терминологии).

ЗАмечаниЕ 1. Стандартная пара Лакса для уравнения синус-Гордон $\varphi, x y=\sin \varphi$ получается при $A=\varphi,{ }_{x}, B=0, C=1, P=0, Q=\sin \varphi$ и $R=-\cos \varphi$. Теорема 1 была сформулирована и доказана Симом для этого случая [6]. 
Рассмотрим наиболее общую линейную задачу $\Psi_{, k}=U_{k} \Psi, k=1,2$, удовлетворяющую лишш следующим условиям:

а) $U_{k}$ - рациональные функции по $\lambda$, имеющие только два полюса при $\lambda=0$ и $\lambda=\infty$;

б) $U_{k}$ представляют собой вешественные линейные комбинации $\mathbf{e}_{1}, \mathbf{e}_{2}$ и $\mathbf{e}_{3}$ (генераторов $s u(2))$;

в) $U_{k}(-\lambda)=\mathbf{e}_{1} U_{k}(\lambda) \mathbf{e}_{1}^{-1}$.

Другими словами,

$$
\Psi, k=\left(a_{k} \mathbf{e}_{1}+\left(b_{k} \lambda+\frac{b_{k}^{\prime}}{\lambda}\right) \mathbf{e}_{2}+\left(c_{k} \lambda+\frac{c_{k}^{\prime}}{\lambda}\right) \mathbf{e}_{3}\right) \Psi
$$

где $a_{k}, b_{k}, b_{k}^{\prime}, c_{k}$ и $c_{k}^{\prime}$-вешественные функции $x^{1}$ и $x^{2}$.

Теорема 2 [5]. Если $\Psi$ удовлетворяет (4), то $F=\Psi^{-1} \Psi, \lambda$ описъвает поверхность постоянной отрицательной гауссовой кривизны $K=-\lambda^{-2}$ в произвольных координатах.

\section{3. ПОВЕРХНОСТИ БИАНКИ}

Пусть $\Psi,,_{k}=U_{k} \Psi$, где $\Psi=\Psi\left(x^{1}, x^{2}, \lambda\right) \in S U(2), U_{1}$ линейно по $\lambda, U_{2}$ линейно по $1 / \lambda$ и $U_{k}(-\lambda)=\mathbf{e}_{1} U_{k}(\lambda) \mathbf{e}_{1}^{-1}$. Другими словами, все предположения те же, что и в случае псевдосферических поверхностей (см. (3)), но теперь спектральная задача "неизоспектральна", т.е. $\lambda=\lambda\left(x^{1}, x^{2}, \zeta\right)$ (на зависимость от координат не накладывается никаких ограничений, кроме дифференцируемости). Отметим, что $\zeta$ теперь является истинным (постоянным!) спектральным параметром. Из условий согласования следует, что

$$
\sigma \lambda^{2}=\frac{2 \zeta g+1}{2 \zeta h-1}
$$

где $h=h\left(x^{1}\right), g=g\left(x^{2}\right)$ и $\sigma=\sigma\left(x^{1}, x^{2}\right)$ не зависят от $\zeta$.

Теорема 3 [5]. Пусть $\Psi$ удовлетворяет задаче (3) $и \lambda=\lambda\left(x^{1}, x^{2}, \zeta\right)$. Тогда $F:=\Psi^{-1} \Psi_{, \zeta}$ описывает поверхность Бианки в $\mathbb{R}^{3}$, а $x^{1}$ и $x^{2}$ являются асимптотическими координатами. Это означает, что гауссова кривизна поверхности $F$ равна $K=-\rho^{-2}$, әде $\rho, 12=0$. Более того, $\rho$ не зависит от $\sigma$ и выполнено соотношение

$$
\rho=\frac{\lambda, \zeta}{\lambda}=\frac{g}{1+2 \zeta g}+\frac{h}{1-2 \zeta h} .
$$

ТЕОРЕМА 4 [5]. Если как $U_{1}$, так и $U_{2}$ являются рациональными функииями по $\lambda$, имеющими два полюса (при $\lambda=0$ и $\lambda=\infty), \quad$ u $\lambda=\lambda\left(x^{1}, x^{2}, \zeta\right)$, то $F:=$ $\Psi^{-1} \Psi, \zeta$ описывает поверхности Бианки в произвольных координатах.

Действительно, прямое выгисление (без каких-либо предположений о виде функции $\lambda$ ) показывает, что $K=-(\lambda / \lambda, \zeta)^{2}$, т.е. $\rho=\lambda, \zeta / \lambda$. Переходя теперь к асимптотическим координатам $x$ и $y$, можно убедиться, что $\rho, x y=0$.

ЗАмечАниЕ 2. Нелинейная система, сопоставляемая поверхностям Бианки, тесно связана с уравнением Эрнста. Поверхности Бианки интенсивно изучались в последние годы (см. [7]). 


\section{4. ОРТОГОНАЛЬНЫЕ ПРЕОБРАЗОВАНИЯ, АЛГЕБРЫ КЛИФФОРДА И ГРУППЫ Spin}

Алгебра Клиффорда $C(N)$ порождается элементами $\mathbf{e}_{1}, \ldots, \mathbf{e}_{N}$ (взаимно ортогональными единичными векторами) при помощи умножения, удовлетворяющего условиям

$$
\mathbf{e}_{j}^{2}=1, \quad \mathbf{e}_{j} \mathbf{e}_{k}=-\mathbf{e}_{k} \mathbf{e}_{j} .
$$

Другими словами, параллельные векторы коммутируют, а ортогональные антикоммутируют по отношению к произведению Клиффорда. В общем случае имеем

$$
v w+w v=2\langle v \mid w\rangle
$$

где угловые скобки обозначают скалярное произведение в векторном пространстве, порождаюшем алгебру Клиффорда.

Пространство $C(N)$ представляет собой $2^{N}$-мерное векторное пространство, состоящее из скаляров, векторов, бивекторов и мультивекторов (включая псевдоскаляр объема $\left.\mathbf{e}_{1} \mathbf{e}_{2} \ldots \mathbf{e}_{N}\right)$. Евклидово пространство $\mathbb{R}^{N}$ можно отождествить с пространством $V$ векторов Клиффорда:

$$
\mathbb{R}^{N} \ni\left(a_{1}, \ldots, a_{N}\right) \longleftrightarrow a_{1} \mathbf{e}_{1}+a_{2} \mathbf{e}_{2}+\cdots+a_{N} \mathbf{e}_{N} \in V \subset C(N) .
$$

Ортогональные преобразования в $\mathbb{R}^{N}$ (т.е. элементы группы $O(N)$ ) можно следуюшим образом выразить с помошью простых операций в алгебре Клиффорда.

ЗАМЕчАнИЕ 3. Известно, что любое ортогональное преобразование (в евклидовом или псевдоевклидовом пространстве) является композицией некоторого числа отражений относительно гиперплоскостей.

Поэтому нам следует начать с формулы

$$
v^{\prime}=-n_{1}^{-1} v n_{1},
$$

где $v^{\prime}$ получается из $v \in V$ при отражении относительно гиперплоскости, ортогональной вектору нормали $n_{1}$. В самом деле, $v=v_{n}+v_{t}$, где $v_{n}$ - компонента, ортогональная к рассматриваемой гиперплоскости, а $v_{t}$ - касательная компонента. Тогда, очевидно, имеем $v^{\prime}=v_{t}-v_{n}$. С другой стороны, нетрудно вычислить, что $n_{1}^{-1} v_{n} n_{1}=v_{n}$ (так как все три вектора коммутируют) и $n_{1}^{-1} v_{t} n_{1}=-v_{t}$ (так как $v_{t} \perp n_{1}$ и, следовательно, они антикоммутируют). Таким образом, формула (5) доказана. Отметим, что вектор $n_{1}^{-1}=n_{1}$ лежит в евклидовом пространстве.

Пусть ортогональное преобразование (или матриша $\Phi \in O(N)$ ) является композицией некоторого числа $M$ отражений относительно гиперплоскостей. Следовательно, его можно представить с помошью последовательности $M$ единичных векторов (векторов нормали к этим гиперплоскостям) $n_{1}, \ldots, n_{M}$. Пусть $\Psi=n_{1} n_{2} \ldots n_{M}$. Тогда

$$
\mathbb{R}^{N} \simeq V \ni v \mapsto(-1)^{M} \Psi^{-1} v \Psi \in V \simeq \mathbb{R}^{N}
$$


очевидным образом является ортогональным преобразованием, порожденным последовательными отражениями относительно гиперплоскостей, ортогональных $n_{1}, \ldots, n_{M}$.

Поэтому удобно ввести группу $\operatorname{Pin}(N)$, состоящую из клиффордовых произведений единичных векторов. Любой элемент $\Psi \in \operatorname{Pin}(N)$ соответствует некоторому ортогональному преобразованию $\Phi \in O(N)$. Так как векторы нормали определены с точностью до знака, то $\Psi$ и $-\Psi$ описывают в точности одно и то же преобразование (в действительности группа $\operatorname{Pin}(N)$ является двулистным накрытием $O(N)$ ).

Аналогичное рассмотрение ортогональных преобразований в псевдоевклидовых пространствах $\mathbb{R}^{p, q}$ приводит к алгебрам Клиффорда $C(p, q)$ и группам $\operatorname{Pin}(p, q)$ (см., например, [8]).

Подгруппа, состоящая из произведений четного числа единичных векторов, называется $\operatorname{Spin}(N)$. Группа $\operatorname{Spin}(N)$ является двулистным накрытием $S O(N)$. Алгеброй Ли группы $\operatorname{Spin}(N)$ служит линейная оболочка бивекторов $\mathbf{e}_{i} \mathbf{e}_{j}, 1 \leqslant i<j \leqslant N$.

ЗАмечаниЕ 4. Спектральные задачи $\Psi, \mu=U_{\mu} \Psi$, где

$$
U_{\mu}=\sum_{j<k} u_{j k} \mathbf{e}_{j} \mathbf{e}_{k}
$$

$\left(u_{j k}\right.$ - (скалярные) вещественные функции $\left.x^{1}, \ldots, x^{m}, \lambda\right)$, являются Spin-значными, т.е. $\Psi$ принимает значения в соответствующей группе $\operatorname{Spin}(N)$.

ПримеР 1. В случае спектральных задач типа ЗШ-АКНС в $s u(2)$ матрицы линейной задачи $U_{1}$ и $U_{2}$ являются линейными комбинациями матриц Паули $i \sigma_{1}, i \sigma_{2}, i \sigma_{3}$. Как известно, они удовлетворяют определяюшим соотношениям алгебры Клиффорда $C(3)$ :

$$
\sigma_{k}^{2}=1, \quad \sigma_{j} \sigma_{k}=-\sigma_{k} \sigma_{j}, \quad j \neq k
$$

Более того,

$$
i \sigma_{1}=\sigma_{2} \sigma_{3}, \quad i \sigma_{2}=\sigma_{3} \sigma_{1}, \quad i \sigma_{3}=\sigma_{1} \sigma_{2}
$$

СлЕдСТВИЕ. Спектральные задачи типа ЗШ-АКНС в sи(2) фактически являются $\operatorname{Spin}(3)$-значными спектральными задачами.

\section{Spin-ЗНАЧНЫЕ СПЕКТРАЛЬНЫЕ ЗАДАЧИ, ЛИНЕЙНЫЕ ПО СПЕКТРАЛЬНОМУ ПАРАМЕТРУ}

Рассмотрим спектральную задачу [4]

$$
\Psi_{, \mu}=U_{\mu} \Psi, \quad \mu=1, \ldots, m, \quad U_{\mu}=\frac{1}{2} \gamma_{\mu}\left(\lambda \mathbf{a}_{\mu}+\mathbf{b}_{\mu}\right)
$$

где $\mathbf{a}_{\mu}=\mathbf{a}_{\mu}\left(x^{1}, \ldots, x^{m}\right) \in W, \mathbf{b}_{\mu}=\mathbf{b}_{\mu}\left(x^{1}, \ldots, x^{m}\right) \in V ; V$ и $W$ - заданные векторные пространства (вложенные в алгебру Клиффорда, порожденную $V \oplus W$; $V \oplus W$ 
имеется скалярное произведение); $\operatorname{dim} W=q$ и $\operatorname{dim} V=r, r \geqslant m ; \gamma_{1}, \ldots, \gamma_{r}$ образуют ортонормированный базис в $V ; \gamma_{r+1}, \ldots, \gamma_{r+q}$ образуют ортонормированный базис в $W ; \Psi$ принимает значения в соответствующей группе Spin. Другими словами,

$$
\mathbf{a}_{\mu}=\sum_{k=1}^{q} \alpha_{\mu k} \gamma_{r+k}, \quad \mathbf{b}_{\mu}=\sum_{k=1}^{r} \beta_{\mu k} \gamma_{k}
$$

где $\alpha_{\mu k}$ и $\beta_{\mu k}$-вешественные скалярные функции $x^{1}, \ldots, x^{m}$

Из условий согласования следует, что $\mathbf{a}_{1}, \ldots, \mathbf{a}_{m}$ образуют ортонормированный репер в $W$ и, следовательно, $m \leqslant q$.

Tеорема 5. Функиия $F=\Psi^{-1} \Psi, \lambda($ вычисленная при $\lambda=0)$ принимает значения в $V \wedge W$.

Для доказательства достаточно найти кинематику ассоциированного репера касательных и нормальных векторов.

ЗАМЕчАниЕ 5. Чтобы получить интересные случаи, обычно используют некоторые специальные проекции $W$ на подходяшие одномерные пространства:

$$
W \mapsto P(W) \subset W, \quad P^{2}=P, \quad \operatorname{dim} P(W)=1 .
$$

Кроме того, необходимо предположить, что $P\left(\Psi^{-1} A \Psi\right)=\Psi^{-1} P(A) \Psi$. Тогда $F$ проецируется на $V \wedge P(W) \simeq V$, т.е. $P$ является погружением $F$ в $V$.

Пример 2. Для изотермических поверхностей в $\mathbb{R}^{n}$ имеем $\operatorname{dim} V=n, \operatorname{dim} W=2$, $W \simeq \mathbb{R}^{1,1}$, a $\operatorname{ker} P$ - любое изотропное направление в $W[4],[9]$.

ПримеР 3. Для ортогональных сетей в $\mathbb{R}^{n}$, удовлетворяюших условию $\sum_{k=1}^{n} H_{k}^{2}=$ const $\left(H_{k}-\right.$ коэффициенты Ламе), имеем $\operatorname{dim} V=\operatorname{dim} W=n$, а $P$ является проекцией на $\gamma_{k}(r+1 \leqslant k \leqslant r+q)[10]$.

ПримеР 4. Для сетей Гишара в $\mathbb{R}^{n}$ (таких ортогональных сетей, что $H_{1}^{2}+H_{2}^{2}=$ $H_{3}^{2}$ ) имеем $\operatorname{dim} V=n, \operatorname{dim} W=3, W \simeq \mathbb{R}^{2,1}$, а ядро проекции $P$ представляет собой “световой фронт" в $W$ (т.е. касательную плоскость к световому конусу в $W$ ).

\section{6. ПРЕОБРАЗОВАНИЕ ДАРБУ-БЕКЛУНДА И ГРУППЫ Spin}

Рассмотрим теперь новую версию стандартного преобразования Дарбу-Беклунда [11]. Вообше говоря, наш подход представляется более или менее эквивалентньм другим имеюшимся методам (см., например, [12]). Однако применительно к обсуждавшейся в предыдушем разделе Spin-значной спектральной задаче этот подход оказывается на удивление простым и весьма удобным.

Наш метод применим к любой спектральной задаче вида (1), т.е. задаче $\Psi, \mu=U_{\mu} \Psi$, $\mu=1, \ldots, m$, где $U_{\mu}$ - матрицы, являюшиеся дробно-рациональными функциями $\lambda$, а $\Psi=\Psi\left(x^{\mu}, \lambda\right)-$ некоторая матрица. 
Как обычно, определим преобразование Дарбу-Беклунда соотношением $\widetilde{\Psi}=D \Psi$, где $D=D\left(x^{1}, \ldots, x^{m}, \lambda\right)$ (в дальнейшем мы будем использовать для краткости обозначение $D=D(\lambda))$. Более того, $\widetilde{\Psi}_{, \mu}=\widetilde{U}_{\mu} \widetilde{\Psi}$, и мы требуем, чтобы матрицы $\widetilde{U}_{\mu}$ и $U_{\mu}$ имели одинаковую структуру и одинаковую зависимость от $\lambda$. Следовательно,

$$
D, \mu+D U_{\mu}=\widetilde{U}_{\mu} D
$$

Предположим теперь, что сушествует такое $\lambda_{1}$, что

$$
D^{2}\left(\lambda_{1}\right)=0
$$

Тогда

$$
D\left(\lambda_{1}\right)_{\mu} D\left(\lambda_{1}\right)+D\left(\lambda_{1}\right) U_{\mu}\left(\lambda_{1}\right) D\left(\lambda_{1}\right)=0 .
$$

Легко проверить, что (8) имеет решение

$$
D\left(\lambda_{1}\right)=\varphi_{1} \Psi\left(\lambda_{1}\right) d_{1} \Psi^{-1}\left(\lambda_{1}\right),
$$

где $\varphi_{1}=\varphi_{1}\left(x^{1}, \ldots, x^{m}\right)$-произвольная скалярная функция, $d_{1}-$ такой постоянный элемент, что $d_{1}^{2}=0$.

Рассмотрим простейшую матрицу Дарбу (т.е. линейную по $\lambda$ матришу $D$ )

$$
D=D_{0}+D_{1} \lambda
$$

Предположим, что имеются два различных значения $\lambda$, например $\lambda_{+}$и $\lambda_{-}$, для которых $D^{2}\left(\lambda_{ \pm}\right)=0$. Тогда

$$
D\left(\lambda_{ \pm}\right)=\varphi_{ \pm} \Psi\left(\lambda_{ \pm}\right) d_{ \pm} \Psi^{-1}\left(\lambda_{ \pm}\right) .
$$

Так как очевидным образом $D\left(\lambda_{ \pm}\right)=D_{0}+D_{1} \lambda_{ \pm}$, то нетрудно выразить $D_{0}$ и $D_{1}$ через $D\left(\lambda_{+}\right)$и $D\left(\lambda_{-}\right)$. В результате находим

$$
D(\lambda)=\frac{\lambda-\lambda_{-}}{\lambda_{+}-\lambda_{-}} \varphi_{+} \Psi\left(\lambda_{+}\right) d_{+} \Psi^{-1}\left(\lambda_{+}\right)+\frac{\lambda-\lambda_{+}}{\lambda_{-}-\lambda_{+}} \varphi_{-} \Psi\left(\lambda_{-}\right) d_{-} \Psi^{-1}\left(\lambda_{-}\right) .
$$

В случае спектральных задач для $(2 \times 2)$-матрищ эта матрица Дарбу эквивалентна односолитонным матрицам Дарбу, полученным другими методами. Вероятно, то же верно для матриц любого порядка. Подробности будут опубликованы в другой статье.

ЗАмечАниЕ 6. Можно заменить матрицы числами Клиффорда. Если $D(\lambda)$ - линейный по $\lambda$ вектор Клиффорда, то $D^{2}(\lambda)$ является скалярной квадратичной по $\lambda$ функцией и уравнение $D^{2}(\lambda)=0$ имеет в точности два решения. Нильпотентный элемент $d_{1}$ является постоянным изотропным вектором Клиффорда. Формулу (9), играюшую ключевую роль при построении матрицы Дарбу, можно интерпретировать как врашение элемента $d_{1}$, выраженное в терминах подходяшей группы Spin. 


\section{7. ПРЕОБРАЗОВАНИЯ ДАРБУ В ТЕРМИНАХ ЧИСЕЛ КЛИФФОР ДА}

Сосредоточимся теперь на спектральной задаче (6)

$$
\Psi_{, \mu}=\frac{1}{2} \gamma_{\mu}\left(\lambda \mathbf{a}_{\mu}+\mathbf{b}_{\mu}\right) \Psi
$$

где $\mathbf{a}_{\mu} \in W$ и $\mathbf{b}_{\mu} \in V, \mu=1, \ldots, m$. Односолитонное преобразование Дарбу для этой спектральной задачи имеет вид [11], [13]

$$
D=\varphi_{0}(\lambda n+\kappa p) \equiv \lambda \hat{n}+\kappa \hat{p},
$$

где

$$
p+i n:=\Psi(i \kappa)\left(p_{0}+i n_{0}\right) \Psi^{-1}(i \kappa),
$$

$p_{0} \in V$ и $n_{0} \in W$ - такие постоянные числа Клиффорда, что $p_{0}^{2}=n_{0}^{2}$, и $\varphi_{0}=1 / \sqrt{p^{2}}$. Заметим, что $n^{2}=p^{2}$ и, следовательно, $\hat{n}^{2}=\hat{p}^{2}=1$.

Простейшее фоновое решение нашей линейной задачи (получаюшееся при $\mathbf{b}_{\mu}=0$ и $\mathbf{a}_{\mu}=f_{\mu}$, где $f_{1}, \ldots, f_{m}$ - постоянный ортонормированный репер в пространстве $W$ ) имеет вид

$$
\Psi_{0}(\lambda)=\exp \left(\sum_{\mu=1}^{m} \frac{1}{2} \lambda \gamma_{\mu} f_{\mu} x^{\mu}\right)=\prod_{\mu=1}^{m} \exp \left(\frac{1}{2} \lambda \gamma_{\mu} f_{\mu} x^{\mu}\right)
$$

(оно факторизуется, поскольку $\left[\gamma_{\mu} f_{\mu}, \gamma_{\nu} f_{\nu}\right]=0$ при $\mu \neq \nu$ ).

В случае изотермических поверхностей, когда $m=2, V$ является линейной оболочкой $\gamma_{1}, \gamma_{2}, \gamma_{3}$, а $W$ - линейной оболочкой $\gamma_{4}, \gamma_{5}\left(\gamma_{1}^{2}=\gamma_{2}^{2}=\gamma_{3}^{2}=\gamma_{4}^{2}=-\gamma_{5}^{2}=1\right)$. Можно также показать, что собственные значения $\lambda_{+}$и $\lambda_{-}$должны иметь вид $\lambda_{ \pm}= \pm i \kappa$, где $\kappa$ вешественно. Поэтому (10) принимает вид

$$
D( \pm i \kappa)=\varphi_{0} \Psi( \pm i \kappa)\left(p_{0} \pm i n_{0}\right) \Psi^{-1}( \pm i \kappa)
$$

где $\varphi_{0}$ - скалярная функция,

$$
p_{0}=p_{01} \gamma_{1}+p_{02} \gamma_{2}+p_{03} \gamma_{3}, \quad n_{0}=n_{04} \gamma_{4}+n_{05} \gamma_{5}
$$

$p_{0 k}, n_{0 \mu}$ - вещественные константы. Положим $f_{1}=\gamma_{5}, f_{2}=\gamma_{4}$ и оценим затравочное решение $\Psi_{0}$ при $\lambda= \pm i \kappa$ :

$$
\Psi_{0}( \pm i \kappa)=\left(\cos \frac{\kappa x}{2} \pm i \gamma_{1} \gamma_{5} \sin \frac{\kappa x}{2}\right)\left(\operatorname{ch} \frac{\kappa y}{2} \pm i \gamma_{2} \gamma_{4} \operatorname{sh} \frac{\kappa y}{2}\right)
$$

где $x:=x^{1}$ и $y:=x^{2}$. Так как $p_{0}+i n_{0}\left(p_{0} \in V, n_{0} \in W\right)$ - изотропный вектор, то $p_{0}$ и $n_{0}$ допускают параметризацию

$$
\begin{gathered}
p_{0}=A_{0}\left(\gamma_{1} \sin \xi_{0}-\gamma_{2} \operatorname{ch} \omega_{0} \operatorname{sh} \tau_{0}-\gamma_{3} \operatorname{sh} \omega_{0}\right), \\
n_{0}=A_{0}\left(\gamma_{4} \operatorname{ch} \omega_{0} \operatorname{ch} \tau_{0}+\gamma_{5} \cos \xi_{0}\right),
\end{gathered}
$$


где $A_{0}$ - константа. Наконец, получаем [11]

$$
D=\lambda \mathbf{n}+\kappa \mathbf{p},
$$

где (в случае затравочного решения $\Psi_{0}$ )

$$
\begin{aligned}
& \mathbf{n}=\frac{\gamma_{4} \operatorname{ch} \omega_{0} \operatorname{ch} \tau+\gamma_{5} \cos \xi}{\sqrt{\operatorname{ch}^{2} \omega_{0} \operatorname{ch}^{2} \tau-\cos ^{2} \xi}} \\
& \mathbf{p}=\frac{\gamma_{1} \sin \xi-\gamma_{2} \operatorname{ch} \omega_{0} \operatorname{sh} \tau-\gamma_{3} \operatorname{sh} \omega_{0}}{\sqrt{\operatorname{ch}^{2} \omega_{0} \operatorname{ch}^{2} \tau-\cos ^{2} \xi}}
\end{aligned}
$$

$\xi:=\kappa x+\xi_{0}$ и $\tau:=\kappa y+\tau_{0}$, что в точности совпадает с нашими прежними результатами [14].

Вычислим теперь двухсолитонное преобразование Дарбу $\widetilde{D}$, получающееся последовательным применением двух преобразований Дарбу вида (12), заданных соответственно параметрами $\kappa_{1}, p_{01}, n_{01}$ и $\kappa_{2}, p_{02}, n_{02}$ :

$$
\widetilde{D}=\left(\lambda \hat{n}+\kappa_{2} \hat{p}\right)\left(\lambda \hat{n}_{1}+\kappa_{1} \hat{p}_{1}\right),
$$

где шляпка означает нормировку (ср. (12)) и

$$
\begin{aligned}
p_{1}+i n_{1} & =\Psi\left(i \kappa_{1}\right)\left(p_{01}+i n_{01}\right) \Psi^{-1}\left(i \kappa_{1}\right), \\
p+i n & =\widetilde{\Psi}\left(i \kappa_{2}\right)\left(p_{02}+i n_{02}\right) \widetilde{\Psi}^{-1}\left(i \kappa_{2}\right), \\
\widetilde{\Psi}\left(i \kappa_{2}\right) & =D\left(i \kappa_{2}\right) \Psi\left(i \kappa_{2}\right)=\left(i \kappa_{2} \hat{n}_{1}+\kappa_{1} \hat{p}_{1}\right) \Psi\left(i \kappa_{2}\right) .
\end{aligned}
$$

После некоторых вычислений приходим к компактной формуле

$$
\widetilde{D}=w(\lambda)+c_{0}\left(\kappa_{1}^{2}-\kappa_{2}^{2}\right) D_{1}(\lambda) \wedge D_{2}(\lambda),
$$

где $c_{0}$ - вещественная скалярная константа, $w(\lambda)$ - вешественный скалярный полином, квадратичный по $\lambda$, а $D_{j}$ порождает односолитонное преобразование (12), параметризованное $\kappa_{j}, p_{0 j}$ и $n_{0 j}$, т.е. $D_{j}=\lambda \hat{n}_{j}+\kappa_{j} \hat{p}_{j}, j=1,2$, и т.д.

Зависимость $c_{0}$ и $w(\lambda)$ от $\kappa_{1}, p_{01}, n_{01}, \kappa_{2}, p_{02}$ и $n_{02}$ весьма сложна (подробности будут скоро опубликованы). Отметим лишь, что, как и следовало ожидать, $c_{0}$ и $w(\lambda)$ симметричны относительно перестановок индексов 1 и 2.

\section{8. ГЕОМЕТРИЧЕСКИЙ СМЫСЛ ТЕОРИИ СОЛИТОНОВ ДЛЯ ЛОКАЛЬНЫХ ПОГРУЖЕНИЙ ПРОСТРАНСТВ ЛОБАЧЕВСКОГО}

Результаты данного раздела основаны на идее Долива и Сантини [15] и были получены совместно с Аминовым [16]. Мы даем геометрическую интерпретацию спектральной задачи, спектрального параметра и формулы Сима для так называемой обобщенной 
системы синус-Гордон [17]

$$
\begin{gathered}
\frac{\partial \alpha_{i k}}{\partial x_{j}}=\alpha_{i j} \beta_{j k}, \quad k \neq j, \\
\frac{\partial \beta_{i k}}{\partial x_{j}}=\beta_{i j} \beta_{j k}, \quad i, j, k \quad \text { попарно различны, } \\
\frac{\partial \beta_{j k}}{\partial x_{j}}+\frac{\partial \beta_{k j}}{\partial x_{k}}+\sum_{i=1}^{n} \beta_{i j} \beta_{i k}=\alpha_{n j} \alpha_{n k}, \quad j \neq k,
\end{gathered}
$$

где индексы $i, j, k=1, \ldots, n$ ( $n \geqslant 2$ фиксировано), $\left(\alpha_{i k}\right)=A$ - ортогональная матрица размера $n \times n, \beta_{j k}$ определены (при $j \neq k$ ) в первом уравнении в (17) и по предположению $\beta_{k k}=0$.

Система (17) неявно описывает $n$-мерные подмногообразия постоянной секционной кривизны $K=-1$ (другими словами, пространства Лобачевского или пространственные формы), погруженные в риманову пространственную форму постоянной кривизны $\widetilde{K}>-1[17]-[19]$. В частности, она описывает погружения $n$-мерного пространства Лобачевского $L^{n}$ кривизны $K=-1$ в евклидово пространство $\mathbb{R}^{2 n-1}$ и в сферы $S^{2 n-1}$ любого радиуса. Спектральная задача со спектральным параметром была впервые поставлена Абловицем, Билсом и Тененблатом [19]. Десять лет спустя к ней была успешно применена формула Сима [20].

Переменные $\beta_{j k}$ алгебраически выражаются через коэффициенты матрицы $A$ и их производные. Это означает, что зависимые переменные системы (17) совпадают с $\alpha_{j k}$ (коэффициенты ортогональной матрицы $A$ заданного размера $n \times n$ ). В случае $n=2$ ортогональная матриша $A$ параметризуется одним углом $\omega$ :

$$
A=\left(\begin{array}{cc}
\cos \omega & \sin \omega \\
-\sin \omega & \cos \omega
\end{array}\right),
$$

и система (17) сводится к уравнению синус-Гордон

$$
\omega, 22-\omega, 11=\sin \omega \cos \omega .
$$

Рассмотрим репер $\Phi \in S O(2 n)$, состояший из $n$ касательных и $n$ нормальных векторов, ассоциированных с погружением $L^{n}$ в сферу $S^{2 n-1} \subset \mathbb{R}^{2 n}$ заданного радиуса $R$. Кинематика движущегося репера дает $n$ линейных матричных уравнений, содержащих параметр $R$ (входящий в виде $R^{-1}$ и $\sqrt{1+R^{-2}}$ ). Выбирая другую (рациональную!) параметризацию

$$
\frac{1}{R}=\frac{1}{2}\left(\frac{1}{\lambda}-\lambda\right), \quad \sqrt{1+\frac{1}{R^{2}}}=\frac{1}{2}\left(\frac{1}{\lambda}+\lambda\right),
$$

мы снова приходим к линейной задаче со спектральным параметром $\lambda$. Переписав эту спектральную задачу в терминах чисел Клиффорда, получаем [16]

$$
\Psi,{ }_{j}=\frac{1}{2} \mathbf{e}_{j}\left(-\sum_{k=1}^{n} \beta_{k j} \mathbf{e}_{k}+\frac{\lambda^{2}+1}{2 \lambda} \sum_{i=1}^{n-1} \alpha_{i j} \mathbf{e}_{n+i}+\frac{\lambda^{2}-1}{2 \lambda} \alpha_{n j} \mathbf{e}_{2 n}\right) \Psi
$$


где $S O(2 n)$-значная величина $\Phi$ заменена $\operatorname{Spin}(2 n)$-значной величиной $\Psi$. Касательные векторы обозначены через $\mathbf{e}_{1}, \ldots, \mathbf{e}_{n}$, а нормальные векторы - через $\mathbf{e}_{n+1}, \ldots, \mathbf{e}_{2 n}$. Последний нормальный вектор $\mathbf{e}_{2 n}$ ортогонален к сфере $S^{2 n-1}$. Следовательно, рассматриваемое погружение (например, $f$ ) $L^{n}$ в $S^{2 n-1}$ можно представить в виде

$$
f=R \Psi^{-1} \mathbf{e}_{2 n} \Psi
$$

Чтобы получить формулу Сима-Тафеля, рассмотрим движение системы отсчета

$$
\widehat{F}:=f-R \mathbf{e}_{2 n}=\frac{2 \lambda}{1-\lambda^{2}}\left(\Psi^{-1}(\lambda) \mathbf{e}_{2 n} \Psi(\lambda)-\mathbf{e}_{2 n}\right)
$$

и перейдем к пределу $R \rightarrow \infty$ (или, что то же самое, $\lambda \rightarrow 1$ ):

$$
F=\lim _{\lambda \rightarrow 1} \widehat{F}=\left.2 \Psi^{-1}(\lambda)\right|_{\lambda=1} \Psi,\left._{\lambda}(\lambda)\right|_{\lambda=1} \mathbf{e}_{2 n}=-\left.2 \mathbf{e}_{2 n} \Psi^{-1} \frac{\partial \Psi}{\partial \lambda}\right|_{\lambda=1} .
$$

Производная $\Psi, \lambda$ возникает после применения правила Лопиталя. Фактор $\mathbf{e}_{2 n}$ коммутирует с $\left.\Psi(\lambda)\right|_{\lambda=1}$ и антикоммутирует с $\Psi,\left._{\lambda}(\lambda)\right|_{\lambda=1}$. Отметим, что он просто проецирует величину $\Psi^{-1} \Psi,_{\lambda}$ (принимающую значения в $\mathbb{R}^{2 n} \wedge \mathbf{e}_{2 n} \simeq \mathbb{R}^{2 n-1}$ ) на $\mathbb{R}^{2 n-1}$.

ЗАмЕчАниЕ 7. Вскоре будет опубликован по крайней мере еще один результат такого рода, касающийся погружений поверхностей постоянной средней кривизны. Будет получено погружение в шестимерное пространство so(4). Для получения поверхности постоянной средней кривизны в $\mathbb{R}^{3}$ необходимо использовать изоморфизм $s o(4) \equiv$ $s o(3) \times s o(3)$. Поиск аналогичных интерпретаций для других интегрируемых систем, имеющих геометрический смысл, и, в частности, для систем, упомянутых в разделе 5 , является интересной открытой проблемой.

Благодарности. Автор признателен Ю. Аминову, В. Бернаки, А. Долива и А. Симу за стимулирующие обсуждения и сотрудничество. Работа поддержана Польским комитетом научных исследований (KBN Грант № 2P03B 126 22).

\section{Список литературы}

[1] A. Sym. Lett. Nuovo Cimento. 1982. V. 33. P. 394-400; 1984. V. 41. P. 353-360.

[2] A. Sym. Soliton surfaces and their application: Soliton geometry from spectral problems. In: Geometric Aspects of the Einstein Equations and Integrable Systems. Lect. Notes Phys. V. 239. Ed. R. Martini. Berlin: Springer, 1985. P. 154-231.

[3] A. I. Bobenko. Surfaces in terms of 2 by 2 matrices: Old and new integrable cases. In: Harmonic Maps and Integrable Systems. Aspects Math. V. 23. Eds. A. P. Fordy, J. C. Wood. Brunswick: Vieweg, 1994. P. 83-128; J. Cieśliński. J. Math. Phys. 1997. V. 38. P. 4255-4272; The Darboux-Bianchi-Bäcklund transformations and soliton surfaces. In: Nonlinearity and Geometry. Eds. D. Wójcik, J. Cieśliński. Warsaw: PWN, 1998. P. 81-107; R. K. Dodd. Commun. Math. Phys. 1998. V. 197. P. 641-665; A.S. Fokas, I. M. Gel'fand, F. Finkel, Q. M. Liu. Sel. Math., New Ser. 2000. V. 6. № 4. P. 347-375.

[4] J. L. Cieśliński. Phys. Lett. A. 2000. V. 267. P. 251-255.

[5] J. L. Cieśliński. J. Phys. A. 2003. V. 36. P. 6423-6440.

[6] A. Sym. Lett. Nuovo Cimento. 1983. V. 36. P. 307-312. 
[7] D. Levi, A. Sym. Phys. Lett. A. 1990. V. 149. P. 381-387; W. K. Schief, C. Rogers, M. E. Johnston. Chaos Solitons Fractals. 1995. V. 5. P. 25-34; J. Tafel. J. Geom. Phys. 1995. V. 17. Р. 381-390; Д. А. Короткин. Зап. научн. семин. ПОМИ. 1996. Т. 234. C. $65-124$.

[8] P. Budinich, A. Trautman. The Spinorial Chessboard. Berlin: Springer, 1988.

[9] J. Cieśliński, P. Goldstein, A. Sym. Phys. Lett. A. 1995. V. 205. P. 37-43.

[10] J. Cieśliński. Adv. Appl. Clifford Algebras. 1997. V. 7. P. 133-139; Rend. Sem. Mat. Messina (Proc. Suppl. Messina Conf. 1998). 2000. P. 135-147.

[11] W. Biernacki, J. L. Cieśliński. Phys. Lett. A. 2001. V. 288. P. 167-172.

[12] В.Е. Захаров, С.В. Манаков, С.П. Новиков, Л. П. Питаевский. Теория солитонов: Метод обратной задачи. М.: Наука, 1980; V. E. Zakharov, A. V. Mikhailov. Commun. Math. Phys. 1980. V. 74. P. 21-40; V. B. Matveev, M. A. Salle. Darboux Transformations and Solitons. Berlin: Springer, 1991; R. Meinel, G. Neugebauer, H. Steudel. Solitonen: Nichtlineare Strukturen. Berlin: Academie, 1991; C.-H. Gu. Bäcklund transformations and Darboux transformations. In: Soliton Theory and Its Applications. Ed. C.-H. Gu. Berlin: Springer, 1995. P. 122-151; J. Cieśliński. J. Math. Phys. 1995. V. 36. P. 5670-5706.

[13] J. L. Cieśliński. J. Phys. A. 2000. V. 33. P. L363-L368.

[14] J. Cieśliński. Differ. Geom. Appl. 1997. V. 7. P. 1-28.

[15] A. Doliwa, P. M. Santini. J. Math. Phys. 1995. V. 36. P. 1259-1273.

[16] J. L. Cieśliński, Yu. A. Aminov. J. Phys. A. 2001. V. 34. P. L153-L159.

[17] K. Tenenblat, C.L. Terng. Ann. Math. 1980. V. 111. P. 477-490.

[18] Ю. А. Аминов. Матем. сб. Нов. сер. 1980. Т. 111(153). С. 402-433.

[19] M. J. Ablowitz, R. Beals, K. Tenenblat. Stud. Appl. Math. 1986. V. 74. P. 177-203.

[20] J. Cieśliński. Phys. Lett. A. 1997. V. 236. P. 425-430. 